hepth/9911138

CALT-68-2248

CITUSC/99-007

\title{
Fivebrane Gravitational Anomalies
}

\author{
Katrin Becker ${ }^{1}$ and Melanie Becker ${ }^{2}$ \\ California Institute of Technology 452-48, Pasadena, CA 91125 \\ and \\ CIT-USC Center for Theoretical Physics, \\ University of Southern California, Los Angeles, CA 90089-2536
}

\begin{abstract}
Freed, Harvey, Minasian and Moore have proposed a mechanism to cancel the gravitational anomaly of the M-theory fivebrane coming from diffeomorphisms acting on the normal bundle. This procedure is based on a modification of the conventional M-theory Chern-Simons term. We compactify this space-time interaction to the ten-dimensional type IIA theory. We then analyze the relation to the anomaly cancellation mechanism for the type IIA fivebrane proposed by Witten.
\end{abstract}

November, 1999

\footnotetext{
1 beckerk@theory.caltech.edu

2 mbecker@theory.caltech.edu
} 


\section{Introduction}

In our journey towards M-theory there appears an object that for a long time has been considered to be rather mysterious, the M-theory fivebrane or M5-brane. The M5-brane was discovered for the first time as a solution to the classical equations of motion of elevendimensional supergravity [1]. The 'fivebrane mystery' originated in part because people did not know how to write down an action for this six-dimensional theory of $(2,0)$ antisymmetric tensor-multiplets. This problem has been solved in [2] and [3] for the case of one single fivebrane. However, the action for $N$ coincident M5-branes is still not known because apparently it is not possible to write down the action for a theory of non-abelian tensormultiplets. For a recent discussion on the subject see [4]. Progress towards understanding this system was made by Harvey, Minasian and Moore [5] who computed the gravitational anomalies of the non-abelian tensor-multiplet theory through anomaly cancellation of a system of $N$ coincident M5-branes. The calculation of [5] is based on the anomaly cancellation mechanism for a single M5-brane proposed by Freed, Harvey, Minasian and Moore (FHMM) [6]. Some earlier attempts to understand anomaly cancellation for the M5-brane were made in [7], [8] and [9].

The perturbative anomaly cancellation for a single fivebrane in type IIA theory was found by Witten [10] who suggested an anomaly cancellation mechanism based on a fivebrane worldvolume counterterm. Since the type IIA theory is dual to M-theory compactified on a circle we expect that both anomaly cancellation mechanisms are related by compactification. It is the purpose of this note to understand the relation between both mechanisms. In section 2 we review some basic ideas which one has to keep in mind for the next sections. In section 3 we will start with the technically simpler case of a chiral string in five dimensions. Here the normal bundle is described by an $S O(3)$ gauge theory. This string originates in compactifications of M-theory on a Calabi-Yau manifold when the M5-brane wraps a non-trivial four-cycle of the Calabi-Yau. It can potentially have gauge and gravitational anomalies. We will show that after compactification to four dimensions the FHMM Chern-Simons term gives a string worldvolume counterterm that cancels the four-dimensional anomaly. This exactly coincides with the lower-dimensional version of the anomaly cancellation mechanism proposed in [10]. In section 4 we consider the M5-brane, 
where the normal bundle is described by an $S O(5)$ gauge theory. The basic idea is the same as in the simpler string example. After compactification to ten dimensions we will obtain the fivebrane worldvolume counterterm of [10]. Our conclusions are presented in section 5 and some useful formulas are collected in an appendix. In this paper we will study perturbative anomalies. The calculation of global anomalies in M-theory still remains an open question.

\section{The Fivebrane Gravitational Anomaly}

The question that was answered in [11], [10], [6] and [5] is whether the low energy effective action of M-theory is well defined even in the presence of extended objects. The extended objects of M-theory are membranes and fivebranes. There are no perturbative anomalies associated with membrane zero-modes because membranes are odd-dimensional and the worldvolume theory is non-chiral. So the only possible anomalies related to membrane zero-modes are global. Their cancellation has been shown in [12]. The situation is very different for the fivebrane because the worldvolume is even-dimensional and the worldvolume theory is chiral. Potentially one could have perturbative anomalies and a calculation is needed to see if these indeed cancel. The M5-brane is a six manifold $W_{6}$ embedded in an eleven manifold. This breaks the Lorentz symmetry to $S O(5,1) \times S O(5)$. For the low energy effective action of M-theory to be well defined, diffeomorphisms which map the fivebrane into itself should be a symmetry of the theory. These are either diffeomorphisms of the fivebrane worldvolume or $S O(5)$ gauge transformations acting on the normal bundle. There are several sources for anomalies. First, there are chiral zero-modes that live on the fivebrane worldvolume. For a single fivebrane these zero-modes form a tensor-multiplet of six-dimensional $(2,0)$ supersymmetry. The chiral fields of this multiplet consist of a chiral fermion transforming in the spinor representation of $S O(5)$ and a two-form potential with anti-self-dual field strength which is a singlet under $S O(5)$. The anomaly can be calculated with the descent formalism. It is expressed in terms of an eightform, $I_{8}=d I_{7}^{(0)}$ and the gauge transformation is given by $\delta I_{7}^{(0)}=d I_{6}^{(1)}$. The anomaly is 
then given by a six-form on the fivebrane worldvolume whose explicit form appears in [10]

$$
2 \pi \int_{W_{6}} I_{6}^{z m(1)}
$$

The second source of anomalies comes from Chern-Simons couplings of the bulk theory

$$
\int_{M_{11}} G_{4} \wedge I_{7}^{b}
$$

Here $G_{4}$ is the four-form field strength and $I_{7}^{b}$ is a gravitational Chern-Simons sevenform that can be expressed in terms of the eleven-dimensional Riemann tensor. This interaction was discovered in [13] by a one-loop calculation in type IIA string theory and in 114 from anomaly cancellation of the tangent bundle. In the presence of fivebranes the gauge invariance of this coupling is broken. Under infinitesimal diffeomorphisms $x^{I} \rightarrow$ $x^{I}+\epsilon v^{I}$ where $\epsilon$ is an infinitesimal parameter and $v$ is a vector field, $I_{7}^{b}$ transforms as $I_{7}^{b} \rightarrow I_{7}^{b}+d I_{6}^{b(1)}$, where $I_{6}^{b(1)}$ is a six-form. Taking the gauge variation of (2.2) and integrating by parts we obtain

$$
\delta S=\int_{M_{11}} d G_{4} \wedge I_{6}^{b(1)}
$$

In the presence of fivebranes the four-form is no longer closed but roughly speaking obeys the Bianchi identity

$$
d G_{4}=2 \pi \delta_{5}
$$

Here $\delta_{5}$ is a five-form which integrates to one in the directions transverse to the fivebrane and has a delta function support on the fivebrane. The total gravitational anomaly was computed in [10] with some standard formulas appearing in [15

$$
2 \pi \int_{W_{6}}\left(I_{6}^{z m}+I_{6}^{b}\right)^{(1)}=2 \pi \int_{W_{6}}\left(\frac{p_{2}(N)}{24}\right)^{(1)} .
$$

Here $p_{2}(N)$ is the second Pontrjagin class of the normal bundle. This is in agreement with the result found in [14] who checked that the anomaly in diffeomorphisms of the tangent bundle cancel.

A new mechanism was needed to cancel the remaining anomaly. Until this point there exist two mechanisms in the literature that do not seem to have an obvious relationship. 
First, Witten [10] introduced a counterterm that lives on the fivebrane worldvolume and that cancels the anomaly in diffeomorphisms of the normal bundle for the ten-dimensional type IIA theory. This mechanism did not seem to have an obvious generalization to M5branes [10]. On the other hand there exists the mechanism proposed in [6] that is useful for M5-branes. It is based on a modification of the eleven-dimensional Chern-Simons term. Here we will show that both mechanisms are not different after all. We will see that the compactification of the modified eleven-dimensional Chern-Simons term of [6] to ten dimensions gives two contributions. First, a modified ten-dimensional Chern-Simons term that is gauge invariant and ensures the compatibility between the ten-dimensional Bianchi identity and the equations of motion. Second, the fivebrane worldvolume counterterm proposed in [10] which cancels the ten-dimensional anomaly. Let us start with the technically simpler case of a chiral string in five dimensions which captures the essence of this calculation.

\section{The Chiral String or $S O(3)$ Gauge Theory}

Consider a compactification of M-theory on a Calabi-Yau threefold. When the M5brane wraps a non-trivial four-cycle of the Calabi-Yau there appears a chiral string in five dimensions that can have both gauge and gravitational anomalies. It was shown in [6] that in five dimensions these anomalies actually cancel. We will show that after compactification to four dimensions the FHMM Chern-Simons term [6] will give at least two contributions. First, a string worldvolume counterterm that cancels the gravitational anomaly coming from diffeomorphisms of the $S O(2)$ normal bundle. This worldvolume counterterm is the lower-dimensional version of the fivebrane worldvolume counterterm found in [10]. Second, a modified four-dimensional Chern-Simons term which is gauge invariant. This will ensure the compatibility between the four-dimensional equations of motion and the corresponding Bianchi identity. Let us see how this works in more detail.

\subsection{The String in Five Dimensions}

This case was worked out in [6] and we will follow closely their discussion. Consider a string in five dimensions located at $y^{i}=0$ for $i=1,2,3$. The theory along the string 
has some properties of gravity coupled to an $S O(3)$ gauge theory. A natural ansatz for the five-dimensional Bianchi identity would be

$$
d G_{2}=\delta\left(y_{1}\right) \delta\left(y_{2}\right) \delta\left(y_{3}\right) d y^{1} \wedge d y^{2} \wedge d y^{3} .
$$

Here $G_{2}$ is the two-form field strength which couples to the stringl. However, the expression (3.1) is not well defined when one is dealing with interactions that are non-linear in the field strength. So for example, order $\delta(0)^{2}$-terms may appear when one is checking the supersymmetry of the Lagrangian. The appearance of $\delta(0)^{2}$-contributions in the Lagrangian was discussed some time ago in [16] for compactifications of M-theory on a manifold with boundary. In fact, there are some striking similarities between both theories. As in the formula above, the Bianchi identity of [16] contains a delta function whose support is at the boundary of space-time. According to [16] the appearance of $\delta(0)^{2}$-terms is a symptom of attempting to treat in classical supergravity what really should be treated in quantum M-theory. In other words, some degrees of freedom are missing in the classical picture.

In the case at hand the authors of [6] made a clever proposal in order to effectively include the missing degrees of freedom. FHMM proposed to smooth out the five-dimensional Bianchi identity by making the following ansatz

$$
d G_{2}=d \rho \wedge \frac{e_{2}}{2} .
$$

The right hand side of this identity is the so-called Thom class of the normal bundle [17]. $\rho=\rho(r)$ is a smooth function of the radial direction away from the string. It takes values $\rho(r)=-1$ for sufficiently small $r$ and $\rho(r)=0$ for sufficiently large $r$. The delta function of (3.1) can be obtained as a limiting function of the bump form $d \rho$. The global angular form $e_{2}$ has integral two over the fiber and is closed

$$
d e_{2}=0
$$

because the Euler class of an odd bundle is zero. The explicit form of $e_{2}$ can be found in the appendix of [6]

$$
e_{2}=\frac{1}{4 \pi} \varepsilon_{a b c}\left(D \hat{y}^{a} D \hat{y}^{b} \hat{y}^{c}-F^{a b} \hat{y}^{c}\right) .
$$

1 Here and in the following we absorb a factor $1 / 2 \pi$ in field strengths and potentials.

2 This corrects a factor 2 in [6]. This factor has to be corrected in the explicit expressions of all angular forms appearing in [6] and [5]. 
Here $\hat{y}^{a}=y^{a} / r$ for $a=1,2,3$ are the coordinates on the fiber. Using the connection $\Theta^{a b}=-\Theta^{b a}$ one can define a covariant derivative

$$
D \hat{y}^{a}=d \hat{y}^{a}-\Theta^{a b} \hat{y}^{b}
$$

and a two-form

$$
F^{a b}=d \Theta^{a b}-\Theta^{a c} \wedge \Theta^{c b}
$$

Formula (3.4) is gauge invariant under the $S O(3)$ gauge transformations generated by $\alpha$

$$
\delta_{\alpha} \Theta^{a b}=(D \alpha)^{a b} \quad \text { and } \quad \delta_{\alpha} \hat{y}^{a}=\alpha^{a b} \hat{y}^{b}
$$

An equivalent formula for $e_{2}$ which will turn out to be useful for explicit calculations is

$$
e_{2}=\frac{1}{4 \pi} \varepsilon_{a b c}\left[d \hat{y}^{a} d \hat{y}^{b} \hat{y}^{c}-d\left(\Theta^{a b} \hat{y}^{c}\right)\right]
$$

which is identical to the expression for $e_{2}$ appearing in 18 that we have collected in the appendix.

Since the angular form is closed and gauge invariant one can apply the descent formalism

$$
e_{2}=d e_{1}^{(0)} \quad \text { and } \quad \delta_{\alpha} e_{1}^{(0)}=d e_{0}^{(1)}
$$

One can now use the above forms to find the solution to the Bianchi identity and the five-dimensional Chern-Simons term. The solution to the Bianchi identity (3.2) which is non-singular on the string is given by

$$
G_{2}=d C_{1}-\frac{1}{2} d \rho \wedge e_{1}^{(0)}
$$

Performing a gauge transformation and demanding that $G_{2}$ should be invariant one obtains that $C_{1}$ transforms under gauge transformations of the normal bundle

$$
\delta_{\alpha} C_{1}=-\frac{1}{2} d \rho e_{0}^{(1)} .
$$

In order to have compatibility between the equations of motion and the Bianchi identity (3.2) one has to correct the space-time action. FHMM proposed that one way of doing this is by introducing a modified Chern-Simons term

$$
S_{C S}=-12 D \pi \lim _{\epsilon \rightarrow 0} \int_{M_{5}-D_{\epsilon}\left(W_{2}\right)}\left(C_{1}-\sigma_{1}\right) \wedge d\left(C_{1}-\sigma_{1}\right) \wedge d\left(C_{1}-\sigma_{1}\right) .
$$


Here the integration is over the five-dimensional space-time without a tubular region of radius $\epsilon$ around the string, $\sigma_{1}=\rho e_{1}^{(0)} / 2$ and $D$ is a constant related to the central charge of the system whose precise definition can be found in [19] and [6].

The Chern-Simons interaction is not gauge invariant because of the gauge transformation

$$
\delta_{\alpha}\left(C_{1}-\sigma_{1}\right)=-\frac{1}{2} d\left(\rho e_{0}^{(1)}\right) .
$$

The variation of the Chern-Simons interaction is then given by a boundary term

$$
\delta_{\alpha} S_{C S}=6 D \pi \lim _{\epsilon \rightarrow 0} \int_{S_{\epsilon}\left(W_{2}\right)} \rho e_{0}^{(1)}\left(G_{2}-\frac{1}{2} \rho e_{2}\right)^{2}
$$

after applying Stoke's theorem. Here one has to use the fact that the boundary of the five-dimensional space without the tubular region $D_{\epsilon}\left(W_{2}\right)$ is an $S^{2}$-sphere bundle over the worldvolume of the string $W_{2}$. This is denoted as $S_{\epsilon}\left(W_{2}\right)$. Since $G_{2}$ and $\rho$ are smooth functions near the brane one obtains in the limit of small $\epsilon$

$$
\delta_{\alpha} S_{C S}=-\frac{3}{2} D \pi \int_{S_{\epsilon}\left(W_{2}\right)} e_{2} e_{2} e_{0}^{(1)}=-3 D \pi \int_{W_{2}} p_{1}^{(1)}(N) .
$$

The relevant formula used to carry out the remaining integration is collected in the appendix. The above anomaly precisely cancels the contributions from chiral zero-modes plus anomaly inflow coming from bulk interactions.

\subsection{Compactification to Four Dimensions}

This is the lower-dimensional counterpart of the fivebrane in ten dimensions that was considered by Witten [10]. The string in four dimensions is described by a one-form field strength and a zero-form potential. The FHMM Bianchi identity for this case is

$$
d H_{1}=\frac{1}{2} d\left(\rho e_{1}\right)
$$

$e_{1}$ is the $S O(2)$ invariant global angular form which satisfies

$$
d e_{1}=2 \chi(F)
$$


where $\chi(F)=\epsilon_{a b} F^{a b} / 4 \pi$ is the Euler class of the $S O(2)$ bundle. From (3.16) we see that the Bianchi identity on the brane is given by

$$
\left.d H_{1}\right|_{W_{2}}=-\chi(F)
$$

which is equivalent to the Bianchi identity used in $[10]^{3}$. The explicit form of $e_{1}$ is

$$
e_{1}=-\frac{1}{\pi} \epsilon_{a b}(D \hat{y})^{a} \hat{y}^{b}=-\frac{1}{\pi} \epsilon_{a b}\left(d \hat{y}^{a} \hat{y}^{b}-\frac{1}{2} \Theta^{a b}\right) .
$$

In order to make contact with the formalism of [10] we can locally write

$$
e_{1} / 2=d \Psi_{0}+\Omega_{1}
$$

where $d \Psi_{0}$ describes the volume term on the fiber and

$$
\Omega_{1}=\frac{1}{4 \pi} \epsilon_{a b} \Theta^{a b}
$$

lives on the string worldvolume. This last quantity is the Chern-Simons one-form in terms of which the Euler character takes the form $\chi(F)=d \Omega_{1}$. Under a gauge transformation we then have

$$
\delta_{\alpha} \Omega_{1}=d \chi_{\alpha} \quad \text { with } \quad \chi_{\alpha}=\frac{1}{4 \pi} \epsilon_{a b} \alpha^{a b}
$$

where $\alpha$ is again the generator of the gauge transformation. Furthermore since $e_{1}$ is gauge invariant we obtain

$$
\delta_{\alpha} \Psi_{0}=-\chi_{\alpha}
$$

Using the forms $\Psi_{0}$ and $\Omega_{1}$ it is easy to find the non-singular solution of the Bianchi identity. It is given by

$$
H_{1}=d B_{0}+\rho \Omega_{1}-d \rho \Psi_{0}
$$

Note that a term of the form $\rho e_{1}$ is not allowed since $e_{1}$ is singular on the brane. To see this note that the integral of $e_{1}$ over the fiber is non-vanishing even if the volume of the

3 In order to relate the present formulation with the one of [10] one has to change the sign of $\Theta$. This explains the minus sign in front of $\chi(F)$ in formula (3.18). 
fiber tends to zero. Since $H_{1}$ should be gauge invariant under the $S O(2)$ transformations of the normal bundle we see that $B_{0}$ has a transformation

$$
\delta_{\alpha} B_{0}=-\rho \chi_{\alpha} .
$$

Let us now work out the relation between the $5 d$ and $4 d$ Chern-Simons terms. For that purpose we would like to consider an $S O(3)$ bundle $N$ of the form $N=N^{\prime} \oplus \mathcal{O}$, where $N^{\prime}$ is an $S O(2)$ bundle and $\mathcal{O}$ is a trivial bundle. We will then assume that the 3 -component of all connections is equal to zero, $\Theta^{3 a}=0$ for $a=1,2$. The expression for $e_{2}$ then becomes

$$
e_{2}=\frac{1}{2 \pi} \omega-\chi(F) \hat{y}^{3}+\frac{1}{4 \pi} \epsilon_{a b} \Theta^{a b} d \hat{y}^{3},
$$

where $\omega$ is the volume element of the $S O(3)$ fiber. Next, notice that the five-dimensional anomaly followed from the Bott and Cattaneo formula

$$
\pi_{*} e_{2}^{3}=2 p_{1}(N)
$$

where $\pi_{*}$ denotes the integration over the fiber. Inserting (3.26) into this expression one obtains after integration over the fiber $p_{1}\left(N^{\prime}\right)=\chi(F)^{2}$, which is the correct relation between the first Pontrjagin class and the Euler class of an even bundle. The formula that FHMM actually used to compute the five-dimensional anomaly is

$$
\pi_{*}\left[e_{2} e_{2} e_{0}^{(1)}\right]=2 p_{1}^{(1)}(N)
$$

In four dimensions the first Pontrjagin class is $p_{1}\left(N^{\prime}\right)=\chi(F)^{2}=d\left(\Omega_{1} \chi(F)\right)$. After a gauge transformation the right hand side of the previous equation becomes $\delta_{\alpha}\left(\Omega_{1} \chi(F)\right)=d\left(\chi_{\alpha} \chi\right)$. This determines $p_{1}^{(1)}\left(N^{\prime}\right)=\chi_{\alpha} \chi(F)$. This is an important piece of information because this together with (3.26) and (3.28) determines the value of $e_{0}^{(1)}$

$$
e_{0}^{(1)}=-\frac{3}{2} \chi_{\alpha} \hat{y}_{3} .
$$

This choice guarantees that the integration over the fiber correctly reproduces the value of $p_{1}^{(1)}\left(N^{\prime}\right)$

$$
\pi_{*}\left[e_{2} e_{2} e_{0}^{(1)}\right]=2 p_{1}^{(1)}\left(N^{\prime}\right)=2 \chi_{\alpha} \chi(F) .
$$


Such a choice is always possible because $e_{1}^{(0)}$ is only determined up to a total derivative. The gauge transformation of the potential appearing in the Chern-Simons term (3.12) is then a total derivative

$$
\delta_{\alpha}\left(C_{1}-\sigma_{1}\right)=-\frac{1}{2} d\left(\rho e_{0}^{(1)}\right)=\frac{3}{4} d\left(\rho \chi_{\alpha} \hat{y}_{3}\right) .
$$

This can be easily expressed in terms of the gauge transformation of the fourdimensional $B_{0}$ field (3.25), so that we can identify

$$
C_{1}-\sigma_{1}=\frac{3}{4} d\left(B_{0} \hat{y}_{3}\right)+\mathcal{I}
$$

where $\mathcal{I}$ represents gauge invariant terms. The anomalous contribution to the ChernSimons term is then given by

$$
S_{C S}^{\mathcal{A}}=\lim _{\epsilon \rightarrow 0}-9 D \pi \int_{M_{5}-D_{\epsilon\left(W_{2}\right)}} d\left(B_{0} \hat{y}_{3}\right)\left(G_{2}-\frac{1}{2} \rho e_{2}\right)\left(G_{2}-\frac{1}{2} \rho e_{2}\right)
$$

Using Stoke's theorem we see that $S_{C S}^{\mathcal{A}}$ is a boundary term

$$
-9 D \pi \lim _{\epsilon \rightarrow 0} \int_{S_{\epsilon}\left(W_{2}\right)} B_{0} \hat{y}_{3}\left(G_{2}-\frac{1}{2} \rho e_{2}\right)\left(G_{2}-\frac{1}{2} \rho e_{2}\right) .
$$

Since the fields $B_{0}$ and $G_{2}$ are smooth near the string we obtain after carrying out the limit and the integration over the fiber

$$
-\frac{9}{4} D \pi \lim _{\epsilon \rightarrow 0} \int_{S_{\epsilon}\left(W_{2}\right)} B_{0} e_{2} e_{2} \hat{y}_{3}=-3 D \pi \int_{W_{2}} B_{0} \chi(F)=3 D \pi \int_{W_{2}} H_{1} \Omega_{1} .
$$

To carry out the integration over the fiber we have used (3.26) and formula (6.6) of the appendix. The result (3.35) is precisely the lower-dimensional analogue of the worldvolume counterterm proposed by Witten for the case of the type IIA fivebrane [10].

In addition there are four-dimensional space-time interactions which are invariant under gauge transformations of the normal bundle. We will not determine invariant terms by a direct calculation because even in $5 d$ the complete set of invariant terms is not known. This would require a microscopic derivation of the interaction presented by FHMM. This derivation is still missing. However, in order to have a consistent $4 d$ theory we expect an additional term in space-time of the form

$$
\int_{M_{4}} C_{1}\left(H_{1}-\frac{1}{2} \rho e_{1}\right) G_{2}
$$


This can be easily seen because once the Bianchi identities are modified $H_{1}$ is no longer closed. Replacing $H_{1}$ by $H_{1}-\rho e_{1} / 2$ guarantees that the Bianchi identities and the equations of motion of this theory are compatible.

\section{The Fivebrane or $S O(5)$ Gauge Theory}

\subsection{The Fivebrane in M-Theory}

The problem of cancelling the anomaly coming from diffeomorphisms of the normal bundle of an M5-brane was solved in [6]. Considering the M5-brane is completely analogous to the chiral string in $5 d$ that we discussed in the previous section. The fivebrane in eleven dimensions breaks the $S O(10,1)$ Lorentz symmetry to $S O(5,1) \times S O(5)$. Therefore the normal bundle is described by an $S O(5)$ gauge theory.

The M5-brane couples to a four-form field strength $G_{4}$ with a three-form potential $C_{3}$. The FHMM Bianchi identity for this case is

$$
d G_{4}=d \rho \wedge \frac{e_{4}}{2} .
$$

The explicit form of $e_{4}$ is given by

$$
\begin{aligned}
e_{4}= & \frac{1}{32 \pi^{2}} \varepsilon_{a_{1} \ldots a_{5}}\left[(D \hat{y})^{a_{1}}(D \hat{y})^{a_{2}}(D \hat{y})^{a_{3}}(D \hat{y})^{a_{4}} \hat{y}^{a_{5}}\right. \\
& \left.-2 F^{a_{1} a_{2}}(D \hat{y})^{a_{3}}(D \hat{y})^{a_{4}} \hat{y}^{a_{5}}+F^{a_{1} a_{2}} F^{a_{3} a_{4}} \hat{y}^{a_{5}}\right],
\end{aligned}
$$

where $a_{i}, i=1, \ldots, 5$ labels the fiber coordinates. One can again apply the descent formalism and introduce the notations

$$
e_{4}=d e_{3}^{(0)} \quad \text { and } \quad \delta e_{3}^{(0)}=d e_{2}^{(1)} .
$$

The eleven-dimensional FHMM Chern-Simons term is

$$
S_{C S}=-\frac{2 \pi}{6} \lim _{\epsilon \rightarrow 0} \int_{M_{11}-D_{\epsilon\left(W_{6}\right)}}\left(C_{3}-\sigma_{3}\right) \wedge d\left(C_{3}-\sigma_{3}\right) \wedge d\left(C_{3}-\sigma_{3}\right),
$$

where $\sigma_{3}=\rho e_{3}^{(0)} / 2$. This term is not invariant under diffeomorphisms. Its variation is obtained by using the anomalous gauge transformation of $C_{3}$ which determines

$$
\delta\left(C_{3}-\sigma_{3}\right)=-d\left(\rho e_{2}^{(1)} / 2\right) .
$$


The result for the gauge transformation of the action is then

$$
\delta S_{C S}=-\frac{\pi}{24} \int_{S_{\epsilon}\left(W_{6}\right)} e_{4} e_{4} e_{2}^{(1)}=-\frac{\pi}{12} \int_{W_{6}} p_{2}^{(1)}(N) .
$$

The last identity can be obtained by using the corresponding version of the formulas by Bott and Catteneo [18]. We have collected the relevant formulas in the appendix.

\subsection{Compactification to Ten Dimensions}

The anomaly cancellation for the fivebrane in type IIA theory has been verified in [10]. In ten dimensions the fivebrane is described by a three-form field strength $H_{3}$ with a two-form potential $B_{2}$. The modified Bianchi identity is

$$
d H_{3}=\frac{1}{2} d\left(\rho e_{3}\right)
$$

The angular form $e_{3}$ is given by

$$
e_{3}=-\frac{1}{2 \pi^{2}} \varepsilon_{a b c d}\left[\frac{1}{3}(D \hat{y})^{a}(D \hat{y})^{b}(D \hat{y})^{c} \hat{y}^{d}-\frac{1}{2} F^{a b}(D \hat{y})^{c} \hat{y}^{d}\right],
$$

where $a=1, \ldots, 4$ labels the $S O(4)$ fiber coordinates. Notice that the sign between both terms is different than in the expression given in [5]. This can be written in the form

$$
e_{3} / 2=d \Psi_{2}+\Omega_{3}
$$

where $\Psi_{2}=\Psi_{2}\left(\hat{y}_{i}, \Theta\right)$ is a function of the fiber and brane coordinates. It contains besides other terms the volume form of $S^{4}$. Its explicit form is not needed in the following. $\Omega_{3}$ is the Chern-Simons three-form

$$
\Omega_{3}=-\frac{1}{32 \pi^{2}} \epsilon_{a b c d}\left(\Theta_{a b} d \Theta_{c d}-\frac{2}{3} \Theta_{a b} \Theta_{c x} \Theta_{x d}\right),
$$

which is related to the Euler class by

$$
\chi(F)=-d \Omega_{3}=\frac{1}{32 \pi^{2}} \varepsilon_{a b c d} F^{a b} \wedge F^{c d} .
$$

Therefore the global angular form $e_{3}$ is related to the Euler class

$$
d e_{3}=-2 \chi(F),
$$


as usual. On the brane we the recover

$$
\left.d H_{3}\right|_{W_{6}}=\chi(F)
$$

which is the Bianchi identity used in [10].

After an $S O(4)$ gauge transformation with generator $\alpha$ the Chern-Simons three-form transforms as a total derivative

$$
\delta_{\alpha} \Omega_{3}=d \chi_{\alpha} \quad \text { with } \quad \chi_{\alpha}=-\frac{1}{32 \pi^{2}} \epsilon_{a b c d} \alpha^{a b} d \Theta^{c d}
$$

In the notation of 10 国 $\Omega_{3}=-\Omega_{\chi}(\Theta)$ and $\chi_{\alpha}=\chi(\alpha, F)$. The invariance of $e_{3}$ implies that $\Psi_{2}$ has a gauge transformation

$$
\delta_{\alpha} \Psi_{2}=-\chi_{\alpha}
$$

In the same way as for the chiral string in $4 d$ there is only one solution of the Bianchi identity which is non-singular on the fivebrane

$$
H_{3}=d B_{2}+\rho \Omega_{3}-d \rho \wedge \Psi_{2}
$$

Demanding $H_{3}$ to be gauge invariant we see that $B_{2}$ must have an anomalous variation under $S O(4)$ transformations

$$
\delta_{\alpha} B_{2}=-\rho \chi_{\alpha}
$$

In order to relate the $11 d$ and $10 d$ theories we will assume that the $S O(5)$ bundle $N$ is of the form $N=N^{\prime}+\mathcal{O}$, where $N^{\prime}$ is an $S O(4)$ bundle and $\mathcal{O}$ is trivial. The connections involving the five-component are then vanishing. Recall that the second Pontrjagin class and the Euler class of the $S O(4)$ bundle are related as $p_{2}\left(N^{\prime}\right)=\chi(F)^{2}$, so that we obtain $p_{2}{ }^{(1)}\left(N^{\prime}\right)=\chi_{\alpha} \chi$. In order to satisfy the Bott and Cattaneo formula

$$
\pi_{*}\left[e_{4} e_{4} e_{2}^{(1)}\right]=2 p_{2}^{(1)}\left(N^{\prime}\right)=2 \chi_{\alpha} \chi(F)
$$

total derivatives for $e_{3}^{(1)}$ have to be chosen in such a way that $e_{2}^{(1)}$ becomes

$$
e_{2}^{(1)}=45 \chi_{\alpha} \hat{y}_{5}
$$

4 There is again a change of sign in $\Theta$. 
To carry out the integration over the fiber we have used the formula (6.11) of the appendix. The $S O(4)$ gauge transformation of the potential appearing in the eleven-dimensional Chern-Simons term is then

$$
\delta_{\alpha}\left(C_{3}-\sigma_{3}\right)=-\frac{1}{2} d\left(\rho e_{2}^{(1)}\right)=-\frac{45}{2} d\left(\rho \chi_{\alpha} \hat{y}_{5}\right) .
$$

The anomalous term of the eleven-dimensional Chern-Simons interaction can be expressed in terms of the ten-dimensional potential $B_{2}$ as

$$
C_{3}-\sigma_{3}=\frac{45}{2} d\left(B_{2} \hat{y}_{5}\right)+\mathcal{I}
$$

where $\mathcal{I}$ is an invariant under $S O(4)$ gauge transformations. Because of the appearance of the total derivative in the previous expression we are able to write the anomalous contribution to the Chern-Simons interaction as a boundary term, exactly as we had done in the lower dimensional case

$$
S_{C S}^{\mathcal{A}}=-\frac{15 \pi}{2} \lim _{\epsilon \rightarrow 0} \int_{S_{\epsilon}\left(W_{6}\right)} \hat{y}_{5} B_{2} \wedge\left(G_{4}-\frac{1}{2} \rho e_{4}\right) \wedge\left(G_{4}-\frac{1}{2} \rho e_{4}\right) .
$$

Since $B_{2}$ and $G_{4}$ are smooth functions near the fivebrane the only nonvanishing contribution to the above integral is

$$
-\frac{15 \pi}{8} \lim _{\epsilon \rightarrow 0} \int_{S_{\epsilon}\left(W_{6}\right)} B_{2} e_{4} e_{4} \hat{y}_{5}
$$

Using formula (6.11) of the appendix we can carry out the integration over the fiber

$$
S_{C S}^{\mathcal{A}}=-\frac{\pi}{12} \int_{W_{6}} B_{2} \chi(F)=\frac{\pi}{12} \int_{W_{6}} H_{3} \Omega_{3} .
$$

This is precisely the counterterm found by Witten 10 that cancels the anomaly from $S O(4)$ transformations of the normal bundle of a type IIA fivebrane.

Of course, as in the lower-dimensional example, in order to have compatibility between the equations of motion and the Bianchi identity (4.7) one should have a modified spacetime Chern-Simons term of the form

$$
\int_{M_{10}} C_{3}\left(H_{3}-\frac{1}{2} \rho e_{3}\right) G_{4} .
$$

However, this does not contribute to the anomaly because it is gauge invariant. It is very satisfying to see that in ten dimensions this anomalous contribution to the space-time interaction can indeed be expressed as a worldvolume counterterm as proposed in [10]. 


\section{Conclusion}

In this paper we have considered a string and a fivebrane embedded in five and eleven dimensions respectively. These theories are not invariant under diffeomorphisms of the normal bundle. This results in an anomaly that can be expressed in terms of the corresponding Pontrjagin class of the normal bundle. Until now there have been two anomaly cancellation mechanisms in the literature whose relation had not been worked out until now. The mechanism proposed by Freed, Harvey, Minasian and Moore [6] is formulated for theories with an odd fiber dimension while the mechanism proposed by Witten [10] is useful for theories with an even fiber dimension. In this paper we saw that both mechanism are actually not different. We have shown that after compactification the FHMM anomaly cancellation mechanism [6] becomes equivalent to the one proposed in [10]. This is very satisfactory and provides further understanding of both anomaly cancellation mechanisms.

Even thought a microscopic derivation of the FHMM Chern-Simons term is still missing we believe that this interaction provides a way of effectively dealing with the presence of $N$ coincident M5-branes. So for example, the proposed interaction explains the $N^{3}$ contributions to the black hole entropy found in [19] from a detailed reduction of the fivebrane tensor-multiplet. The $N^{3}$ growth of the entropy of a system of multiple M5-branes was first discovered in [20].

The microscopic description of $N$ coincident fivebranes is a theory of non-abelian tensor-multiplets. Such a theory does not seem to exist. At least not as a local quantum field theory [4]. So the only possibility seems to be a non-local theory. At this point our most convincing candidate to be a satisfactory formulation of M-theory is Matrix theory [21]. However, it is very hard to describe M5-branes in this approach. It is rather possible that the final formulation of M-theory may involve a non-local theory. Before trying to answer this more difficult question it would be important to find a global formulation of the mechanism proposed by FHMM. It seems plausible that gerbes [22] might be the correct framework to address this question. Furthermore, Freed and Witten have computed global anomalies for type II theories [23]. Maybe an extension of this work to the elevendimensional M-theory is possible. We hope to report on this in a future. 


\section{Acknowledgement}

We are grateful to Jeff Harvey, Petr Horava, Greg Moore, John Schwarz and Edward Witten for useful discussions. This work was supported by the U.S. Department of Energy under grant DE-FG03-92-ER40701.

\section{Appendix}

In this appendix we would like to collect some formulas that are useful when computing the integrals involved in the calculation of the anomalies presented in this paper. We would like to begin with integrals involving $e_{2}$. First $e_{2}$ can be rewritten in the form

$$
e_{2}=\frac{\omega+d\left(\theta^{c} \hat{y}_{c}\right)}{2 \pi}
$$

where $\omega$ is the volume element

$$
\omega=\hat{y}_{1} d \hat{y}_{2} d \hat{y}_{3}+\hat{y}_{2} d \hat{y}_{3} d \hat{y}_{1}+\hat{y}_{3} d \hat{y}_{1} d \hat{y}_{2}
$$

which satisfies $\int_{S^{2}} \omega=4 \pi$ and $\theta_{c}=-\epsilon_{a b c} \Theta^{a b} / 2$. This expression implies

$$
\pi_{*} e_{2}=2
$$

Where by $\pi_{*}$ we denote the integration over the fiber. Moreover,

$$
\begin{aligned}
(2 \pi)^{2} \pi_{*} e_{2}^{2}= & \pi_{*}\left[2 \omega d\left(\theta^{a} \hat{y}_{a}\right)+d\left(\theta^{a} \hat{y}_{a}\right) d\left(\theta^{b} \hat{y}_{b}\right)\right]= \\
& 2 d \theta^{a} \pi_{*}\left(w \hat{y}_{a}\right)-\theta^{a} \theta^{b} \pi_{*}\left(d \hat{y}_{a} d \hat{y}_{b}\right)=0 .
\end{aligned}
$$

The explicit evaluation of the Bott and Cattaneo formula goes along the same lines [18]

$$
\begin{aligned}
(2 \pi)^{3} \pi_{*} e_{2}^{3}= & \pi_{*}\left[3 \omega d\left(\theta^{a} \hat{y}_{a}\right)^{2}+d\left(\theta^{a} \hat{y}_{a}\right)^{3}\right]= \\
& 3 d \theta^{a} d \theta^{b} \pi_{*}\left(\omega \hat{y}_{a} \hat{y}_{b}\right)-3 d \theta^{a} \theta^{b} \theta^{c} \pi_{*}\left(\hat{y}_{a} d \hat{y}_{b} d \hat{y}_{c}\right) .
\end{aligned}
$$

Introducing spherical coordinates it is the easy to see that

$$
\pi_{*}\left(\omega \hat{y}_{a} \hat{y}_{b}\right)=\frac{4}{3} \pi \delta_{a b} \quad \text { and } \quad \pi_{*}\left(\hat{y}_{a} d \hat{y}_{b} d \hat{y}_{c}\right)=\frac{4}{3} \pi \varepsilon_{a b c} .
$$

Therefore we obtain the equation

$$
\pi_{*} e_{2}^{3}=\frac{1}{2 \pi^{2}}\left(d \theta^{a} d \theta_{a}-\varepsilon_{a b c} d \theta^{a} \theta^{b} \theta^{c}\right)=2 p_{1} .
$$


In general $p_{r}$ denote the Pontrjagin classes.

The evaluation of these expressions for the $S O(5)$ case is, of course, more involved but in principle straightforward. Without repeating the same steps as for the $S O(3)$ case we just state here the results

$$
\pi_{*} e_{4}=2, \quad \pi_{*} e_{4}^{2}=0 \quad \text { and } \quad \pi_{*} e_{4}^{3}=2 p_{2} .
$$

If $\omega$ now denotes the volume form of the $S^{4}$ fiber

$$
\omega=\frac{1}{4 !} \epsilon_{a_{1} a_{2} a_{3} a_{4} a_{5}} d \hat{y}^{a_{1}} d \hat{y}^{a_{2}} d \hat{y}^{a_{3}} d \hat{y}^{a_{4}} \hat{y}^{a_{5}}
$$

with $\int_{S^{4}} \omega=8 \pi^{2} / 3$, then

$$
\pi_{*}\left(\omega \hat{y}_{a} \hat{y}_{b}\right)=\frac{8}{15} \pi^{2} \delta_{a b}
$$

This equation implies that after imposing the condition that the 5-component of the connection vanishes we have

$$
\pi_{*}\left(e_{4} e_{4} \hat{y}^{5}\right)=\frac{2}{45} \chi(F)
$$

as a simple evaluation of the involved integrals implies. 


\section{References}

[1] R. Güven, "Black p-Brane Solutions of $D=11$ Supergravity Theory", Phys. Lett. B276 (1992) 49.

[2] P. Pasti, D. Sorokin and M. Tonin, "Covariant Action for a $D=11$ Five-Brane with the Chiral Field", Phys. Lett. B398 (1997) 41, hep-th/9701037, I. Bandos, K. Lechner, A. Nurmagambetov, P. Pasti, D. Sorokin and M. Tonin, "Covariant Action for the Superfive-Brane of M Theory", Phys. Rev. Lett.78 (1997) 4332, hep-th/9701149.

[3] M. Aganagic, J. Park, C. Popescu and J. Schwarz, "Worldvolume Action for the MTheory Fivebrane", Nucl. Phys. B496 (1997) 191, hep-th/9701166.

[4] X. Bekaert, M. Henneaux and A. Sevrin, "Deformations odd Chiral Two-Forms in Six Dimensions", hep-th/9909094.

[5] J. A. Harvey, R. Minasian and G. Moore, "Nonabelian Tensor Multiplet Anomalies", JHEP 9809:004, (1998), hep-th/9808060.

[6] D. Freed, J. A. Harvey, R. Minasian and G. Moore, "Gravitational Anomaly Cancellation for M Theory Fivebranes", Adv. Theor. Math. Phys. 2 (1998) 601, hepth/9803205.

[7] S. P. de Alwis, "Coupling of Branes and Normalization of Effective Actions in String/M Theory", Phys. Rev. D56 (1997) 7963, hep-th/9705139.

[8] L. Bonora, C. S. Chu and M. Rinaldi, "Perturbative Anomalies of the M-5-Brane", hep-th/9710063; "Anomalies and Locality in Field Theories and M-Theory", hepth/9712205.

[9] M. Henningson, "Global Anomalies in M Theory", Nucl. Phys. B515 (1998) 233, hep-th/9710126.

[10] E. Witten, "Five-Brane Effective Action in M-Theory", J. Geom. Phys. 22 (1997) 103, hep-th/9610234.

[11] E. Witten, "Five-Branes and M-Theory on an Orbifold", hep-th/9512219.

[12] E. Witten, "Flux Quantization in M-theory", J. Geom. Phys. 22 (1997) 1, hepth/9609122.

[13] C. Vafa and E. Witten, "A One-Loop Test of String Duality", Nucl. Phys. B447 (1995) 261, hep-th/9505053.

[14] M. J. . Duff, J. T. Liu and R. Minasian, "Eleven- Dimensional Origin of String/String Duality: A One Loop Test", Nucl. Phys. B452 (1995) 261, hep-th/9509084.

[15] L. Alvarez-Gaumé and E. Witten, "Gravitational Anomalies", Nucl. Phys. B234 (1983) 269.

[16] P. Horava and E. Witten, "Eleven-Dimensional Supergravity on a Manifold with Boundary", Nucl. Phys. B475 (1996) 94.

[17] R. Bott and L. W. Tu, "Differential Form in Algebraic Topology", Springer-Verlag, 1982, New York. 
[18] R. Bott and A. S. Cattaneo, "Integral invariants of 3-Manifolds", dg-ga/9710001.

[19] J. Maldacena, A. Strominger and E. Witten, "Black Hole Entropy in M -theory", hep-th/9711053.

[20] I. R. Klebanov and A. Tseytlin, "Entropy of Near Extremal P-Branes", Nucl. Phys. B475 (1996) 164, hep-th/9604089.

[21] T. Banks, W. Fischler, S. H. Shenker and L. Susskind, "M Theory as a Matrix Model: A Conjecture", Phys. Rev. D55 (1997) 5112.

[22] N. Hitchin, "Lectures on Special Lagrangian Submanifolds", math.dg/9907034.

[23] D. S. Freed and E. Witten, "Anomalies in String Theory with D-Branes", hepth/9907189. 